\title{
1,3-Dibromo-5,5-dimethylhydantoin as a Novel Oxidizing Agent for the Oxidation of 1,3,5-Trisubstituted Pyrazolines under Both Heterogeneous and Solvent-free Conditions
}

\author{
Davood Azarifar, Mohammad Ali Zolfigol," and Behrooz Maleki \\ Chemstry Department, College of Science, Bu-Ali Sina Universitvi, Hamadan 65174, Iran \\ Received July 9, 2003
}

Key Words : 1.3-Dibromo-5,5-dimethylhydantoin (DBH), 1.3,5-Trisubstituted pyrazolines. Pyrazoles. Solvent-free conditions

Five-membered heterocyclic compounds are stable aromatic compounds and have been used commercially as phamaceuticals. pesticides. and dyestuffs. These compounds are also important constituents that often exist in biologically: active natural products and synthetic compounds of medicunal interest. ${ }^{1: 2}$ Conversion of 1.3.5-trisubstituted pyrazolines with sensitive functional groups to their corresponding pyrazoles derivatives is a tricky step. Although a variety of reagents such as $\mathrm{Pd} / \mathrm{C} / \mathrm{acetic}$ acid. ${ }^{3}$ acid cobalt soap of fatty acids. ${ }^{4}$ lead tetraacetate ${ }^{5}$ mercury oxide. ${ }^{6}$ manganese dioxide. ${ }^{7}$ potassium permangenate ${ }^{8}$ silver nitrate. ${ }^{9}$ 1odobenzene diacetate ${ }^{10}$ zirconium nutrate ${ }^{11}$ are capable of affecting the pyrazolines oxidation. this transformation remains capricious because these compounds are very sensitive to the oxidizing agents and reaction conditions. Moreover most of the reported reagents produce some byproducts. which etther destroy or are difficult to remove from the sensitive pyrazoles. ${ }^{+}$Another major drawback to the older procedures is their use of reagents which are either highly toxic or produce serious disposal problems (or both).

Our goal in undertaking this line of work was three-fold: a) to overcome the limitations and drawbacks of the reported methods such as tedious work-up. acidic media. ${ }^{3}$ and safety problems (presence of toxic transition metal cations such as $\mathrm{Co}(\mathrm{II}),{ }^{4} \mathrm{~Pb}(\mathrm{IV}){ }^{5} \mathrm{Hg}(\mathrm{II}) .{ }^{6} \mathrm{Mn}(\mathrm{IV}$ and $\mathrm{VII}) .{ }^{7.8} \mathrm{Ag}(\mathrm{I}){ }^{9} \mathrm{Zr}(\mathrm{IV})^{11}$ within molecular structure of the reagents): (b) solvent-free organic sy'nthesis seems to be a highly useful technique. especially for industry and it has many advantages: reduced pollution. low costs. and simplicity in process and handlung (these factors are especially important in industry) ${ }^{13-14}$ (c) moreover. to develop a high-yielding synthesis of pyrazoles by using a novel commercially avalable reagent. Therefore. we were interested to find a heterogeneous system for pyrazoline oxidation. In continuation of our studies in this regard. ${ }^{l}$ we have found that DBH a cheap commercially avalable reagent. which recently used as an excellent reagent for analytical purposes ${ }^{15-90}$ has found little application in organic chemistry ${ }^{-1-27}$ Therefore we wish to report a simple. cheap and convenient method for the effective conversion of 1.3.5-trisubstituted pyrazolines (1) to their corresponding pyrazoles (2) by using DBH both under

\footnotetext{
Corresponding author. E-mail: Zolfi tabasuac.ir
}

\begin{tabular}{|c|c|c|c|c|}
\hline \multicolumn{2}{|l|}{ Scheme 1} & & \multirow{4}{*}{$\begin{array}{r}\mathrm{R}^{2} \\
\frac{2}{\mathrm{~N}_{-\mathrm{N}}} \\
\mathrm{R}^{3}\end{array}$} \\
\hline $\mathrm{R}^{2}$ & & & & \\
\hline \multicolumn{2}{|l|}{1} & \multicolumn{2}{|c|}{ Solid phase } & \\
\hline Substrate & Product & $\mathrm{R}^{1}$ & $\mathrm{R}^{2}$ & \\
\hline $1 \mathrm{a}$ & $2 a$ & $\mathrm{Ph}$ & 2-Naphthyl & $2-\mathrm{CH}_{3} \mathrm{C}_{4} \mathrm{H}_{4}$ \\
\hline $1 b$ & $2 b$ & $\mathrm{Ph}$ & 2-Naphthyl & Ph \\
\hline $1 c$ & $2 c$ & $\mathrm{Ph}$ & $\mathrm{Ph}$ & Ph \\
\hline $1 d$ & 2d & $\mathrm{Ph}$ & $4-\mathrm{CH}_{3} \mathrm{C}_{4} \mathrm{H}_{4}$ & $3-\mathrm{CH}_{3} \mathrm{C}_{6} \mathrm{H}_{4}$ \\
\hline 1e & $2 e$ & $\mathrm{Ph}$ & $3-\mathrm{CH}_{3} \mathrm{C}_{6} \mathrm{H}_{4}$ & $2-\mathrm{ClC}_{6} \mathrm{H}_{4}$ \\
\hline 1f & $2 f$ & $\mathrm{Ph}$ & $4-\mathrm{CH}_{3} \mathrm{OC}_{6} \mathrm{H}_{4}$ & $3-\mathrm{CH}_{3} \mathrm{C}_{6} \mathrm{H}_{4}$ \\
\hline 1g & $2 \mathrm{~g}$ & $\mathrm{Ph}$ & $4-\mathrm{CH}_{3} \mathrm{OC}_{6} \mathrm{H}_{4}$ & $2-\mathrm{CH}_{3} \mathrm{C}_{6} \mathrm{H}_{4}$ \\
\hline $1 \mathrm{~h}$ & $2 \mathrm{~h}$ & $\mathrm{Ph}$ & $4-\mathrm{CH}_{3} \mathrm{OC}_{6} \mathrm{H}_{4}$ & Ph \\
\hline $1 \mathbf{i}$ & $2 \mathbf{i}$ & $\mathrm{Ph}$ & $4-\mathrm{CH}_{3} \mathrm{OC}{ }_{6} \mathrm{H}_{4}$ & $4-\mathrm{ClC}_{6} \mathrm{H}_{4}$ \\
\hline $1 \mathbf{j}$ & $2 \mathbf{j}$ & $\mathrm{Ph}$ & $3-\mathrm{CH}_{3} \mathrm{C}_{6} \mathrm{H}_{4}$ & $4-\mathrm{ClC}_{6} \mathrm{H}_{4}$ \\
\hline $1 \mathrm{k}$ & $2 k$ & $\mathrm{Ph}$ & 2-Naphthyl & $3-\mathrm{CH}_{3} \mathrm{C}_{6} \mathrm{H}_{4}$ \\
\hline 11 & 21 & $\mathrm{Ph}$ & 2-Naphthyl & $4-\mathrm{ClC}_{6} \mathrm{H}_{4}$ \\
\hline $1 \mathrm{~m}$ & $2 \mathrm{~m}$ & $\mathrm{Ph}$ & 2-Naphthỵl & $2-\mathrm{ClC}_{+} \mathrm{H}_{4}$ \\
\hline 1n & $2 n$ & Ph & $3-\mathrm{CH}_{3} \mathrm{C}_{5} \mathrm{H}_{4}$ & $4-\left(\mathrm{CH}_{3}\right)_{2} \mathrm{NC}_{4} \mathrm{H}_{4}$ \\
\hline 10 & 20 & Ph & $2-\mathrm{CH}_{3} \mathrm{C}_{5} \mathrm{H}_{4}$ & $4-\left(\mathrm{CH}_{3}\right)_{2} \mathrm{NC}_{3} \mathrm{H}_{4}$ \\
\hline $1 p$ & $2 p$ & Ph & $4-\mathrm{CH}_{3} \mathrm{OC}_{6} \mathrm{H}_{4}$ & $2-\mathrm{ClC}_{+} \mathrm{H}_{4}$ \\
\hline $1 q$ & $2 q$ & $\mathrm{COCH}_{3}$ & 2-Naphthyl & $2-\mathrm{CH}_{3} \mathrm{C}_{6} \mathrm{H}_{4}$ \\
\hline $1 \mathbf{r}$ & $2 \mathbf{r}$ & $\mathrm{COCH}_{3}$ & 2-Naphthyl & $3-\mathrm{CH}_{3} \mathrm{C}_{6} \mathrm{H}_{4}$ \\
\hline $1 s$ & $2 s$ & $\mathrm{COCH}_{3}$ & $3-\mathrm{CH}_{3} \mathrm{C}_{6} \mathrm{H}_{4}$ & $2-\mathrm{ClC}_{2} \mathrm{H}_{4}$ \\
\hline $1 \mathrm{t}$ & $2 t$ & $\mathrm{COCH}_{3}$ & $2-\mathrm{CH}_{3} \mathrm{C}_{4} \mathrm{H}_{4}$ & $2-\mathrm{ClC}_{6} \mathrm{H}_{4}$ \\
\hline $1 \mathrm{u}$ & $2 u$ & $\mathrm{CH}_{3} \mathrm{CH}_{2}$ & $\mathrm{Ph}$ & Ph \\
\hline
\end{tabular}

heterogeneous and also solvent free-conditions (Scheme 1).

Different kinds of 1.3.5-trisubstituted pyrazolines were subjected to oxidation reaction in the presence of $\mathrm{DBH}$ in $\mathrm{CCl}_{4}$ (Scheme 1) or solvent-free conditions. The oxidation reactions were performed under mild conditions at room temperature with good yields. The 1.3.5-trisubstituted pyrazoles (2) can be obtained by simple filtration and evaporation of the solvent. ${ }^{28-30}$ The results and reaction conditions are given in the Table. N-Bromosuccinimide was also used as oxidizing agent under same condition (Entries 2 and 4). 
Table 1. Oxidation of 1,3,5-trisubstituted prrazolines (1) to their corresponding pyrazoles (2) with 1,3-Dibronno-5,5-dimethylhydantoin (DBH) or N-bromosuccininide (NBS) both in carbon tetrachloride $(\mathbf{I})$ and solvent-free conditions (II) at room temperature

\begin{tabular}{|c|c|c|c|c|c|c|}
\hline \multirow{2}{*}{ Entry } & \multirow{2}{*}{$\begin{array}{l}\text { Sub- } \\
\text { strate }\end{array}$} & \multirow{2}{*}{$\begin{array}{l}\text { Prod- } \\
\text { uct }\end{array}$} & \multicolumn{2}{|c|}{ Reagent/Substrate } & \multirow{2}{*}{$\frac{\text { Tinne (h) }}{\mathbf{I}(\mathbf{I})}$} & \multirow{2}{*}{$\frac{\text { Yield }^{c}(\%)}{\mathbf{I}(\mathbf{I})}$} \\
\hline & & & I & II & & \\
\hline 1 & 1a & $2 a$ & 1 & 2.5 & $0.3(1)$ & $94(85)$ \\
\hline 2 & $1 \mathbf{a}$ & $2 a$ & $1.25^{7}$ & $4^{d}$ & $0.3^{i}(1)^{i}$ & $80^{7}(72)^{7}$ \\
\hline 3 & lb & $2 b$ & 1 & 2.25 & $0.3(1.25)$ & $98(90)$ \\
\hline 4 & lb & $2 b$ & $1.25^{a}$ & $4^{d}$ & $0.3^{z^{i}}(1.25)^{7^{7}}$ & $85^{i}(75)^{7}$ \\
\hline 5 & $1 \mathrm{c}$ & $2 c$ & 1 & 2.25 & $0.5(1.25)$ & $90(83)$ \\
\hline 6 & 1d & $2 d$ & 1.5 & 3.75 & $0.5(1.5)$ & $93(87)$ \\
\hline 7 & le & $2 \mathrm{e}$ & 2.25 & 3.5 & $0.3(0.75)$ & $98(90)$ \\
\hline 8 & If & $2 f$ & 1.75 & 3 & $0.5(1)$ & $95(90)$ \\
\hline 9 & 19 & $2 \mathrm{~g}$ & 1.75 & 3.5 & $0.5(1)$ & $90(85)$ \\
\hline 10 & lh & $2 \mathrm{~h}$ & 2 & 3.5 & $0.3(1)$ & $70(63)$ \\
\hline 11 & li & $2 \mathrm{i}$ & 1.25 & 3.75 & $0.5(1.5)$ & $81(76)$ \\
\hline 12 & $\mathbf{1 j}$ & $2 \mathbf{j}$ & 2.25 & 4 & $0.75(1.5)$ & $96(92)$ \\
\hline 13 & $1 \mathrm{k}$ & $2 \mathrm{k}$ & 2.25 & 3.75 & $0.75(1.25)$ & $98(91)$ \\
\hline 14 & 11 & 21 & 2.5 & 3.75 & $0.5(1.25)$ & $92(87)$ \\
\hline 15 & $1 \mathrm{~m}$ & $2 \mathrm{~m}$ & 2.25 & 3.5 & $0.5(1)$ & $85(74)$ \\
\hline 16 & $\ln$ & $2 n$ & 2 & 3.25 & $0.3(0.75)$ & $88(76)$ \\
\hline 17 & 10 & 20 & 2 & 3 & $0.3(0.75)$ & $92(85)$ \\
\hline 18 & 1p & $2 p$ & 2 & 3.5 & $0.3(1)$ & $90(82)$ \\
\hline 19 & 19 & $2 q$ & 3.5 & 14 & $1(2.25)$ & $94(86)^{e}$ \\
\hline 20 & $1 \mathrm{r}$ & $2 r$ & 3.5 & 15 & $0.75(3)$ & $92(89)^{e}$ \\
\hline 21 & $1 s$ & $2 s$ & 4.25 & 18 & $1.75(4.5)$ & $90(72)^{e}$ \\
\hline 22 & lt & $2 \mathrm{t}$ & 4 & 15 & $1.75(2.5)$ & $93(80)^{e}$ \\
\hline 23 & 1u & $2 u$ & 1 & 2 & $0.15(0.3)$ & $90(80)$ \\
\hline
\end{tabular}

"All of the isolated products are biown compounds and their spectra and physical data have been reported in the literature. ${ }^{\hat{-}-11}{ }^{k}$ Molar ratio of $1,3-$ Dibromo-5.5-dimethylhydntoin (DBH) in $\mathrm{CCl}_{4}$ (I) $\mathrm{mmol}$ and under solvent free conditions (II) mmol. "Isolated vields. "K-Bromosuccinimide (NBS) was used as oxidizing agent. "These reactions were occurred at $80^{\circ} \mathrm{C}$ under solvent free conditions.

In conclusion. practical and efficient oxidations of 1.3.5trisubstituted pyrazolines have been achieved by the new methodology described. Thus this reagent could be used for the oxidation of a wide variety of pyrazolines derivatives under safe condition.

Acknowledgment. Financial support for this work by the Research Council of Bu-Ali Sina University. Hamadan. Iran. is gratefully acknowledged.

\section{References}

1. Azarifar. D.: Shaebanzadeh. M. Molecules 2002. 7.885.

2. Gilchrist. T. L. Heterocvclic Chemishy. 2nd Ed: John Willey \& Sons. Inc.: New York. 1992: p 294.

3. Nakamichi. N.: Kawashita. Y.: Hayashi. M. Org. Lett. 2002. t. 3955 .
4. Shah. J. N.: Shah. C. K. J. Org. Chem. 1978. 43.1266.

5. Goldstone. W. A. F.: Norman. R. O. C. d. Chem Soc. Chem. Contmint 1966. 1536.

6. Alwers. K: Heimke. P. Liebigs Am. 1927. 458.186.

7. Bhatnagar. I.: George. M. V. Tetwathedron 1968. 24. 1293.

8. Smith. L. I.: Howard, K. L. J. Am Chem. Soc. 1943. 65. 159.

9. Dodwadmath, R. P. Wheeler. T. S. Proc Ind Acad Sci. 1935, 2 A. 438

10. Singh. S. P.: Kumar. D: Prakash. O.: Kapoor. R. P. Sinth. Conmum. 1997.27.2683.

11. Sabitha. G.; Kumar Reddy: G. S. K.: Reddy: Ch. S.; Fatima, N. Yadav. J. S. Synthesis $\mathbf{2 0 0 3}, 1267$.

12. Vamma. R. S. Green Chem. 1999, 1.43.

13. Tanaka. K.: Toda. F. Chem. Rev 2000. 100. 1025.

14. Krehn1ak. V: Holladay. M. W. Chem. Rev: 2002. 102.61.

15. Hilp. M.: Senjuk. S. J. Pharnt. Bioned Anal 2001. 25. 363.

16. Hilp. M. Phanmazie 2001, 56,777

17. Hilp. M. Phamazie 2002, $57,45$.

18. Hilp. M. Phamazie 2002, 57,316.

19. Hilp. M. J. Pham Biomed Anal 2002. 28. 303.

20. Hilp. M. J. Pham Bioned Anal 2002. 28.337.

21. Walter. T. R.: Zajac. W. W.: Woods. J. M. J. Org. Chem. 1991.56. 1316 .

22. Uchibori, Y; Umeno, M.; Seto. H.: Yoshioka. I. Chem. Lett 1993. 4. 673

23. Shimizu. M.: Nakahara. Y: Yoshioka. H. J. Chem. Soc. Chem. Conmum. 1989.24.1881

24. Sondej. S. C.: Katzenellenbogen. T. A. J. Org. Chent 1986. 51. 3508 .

25. Shimizu. M.; Nakahara, Y.: Yoshioka. H. J. Fluorine Chem. 1999. 97.57.

26. Chassaing. C.: Haudrechy. A.: Langlois. Y. Tetrdhedron Lett. 1997.38. 4415

27. Furuta. S.: Hiyama. T. Tetrahedron Lett. 1996. 37.7983

28. Chemicals were purchased from Fluka, Merck. Riedel-dehaen AG and Aldrich chemical companies. Ylelds refer to isolated pure products. The oxidation products were characterized by comparison of their spectral (IR. and ${ }^{1} \mathrm{H}-\mathrm{NMR}$.) and physical data with the authentic samples. ${ }^{3.11}$ All 1.3.5-trisubstituted pyrazolines were synthesized according to our previously reported procedure. ${ }^{3}$

29. General procedure for ovidation of pyrazoline in solution: A suspension of $\mathrm{DBH}$ (The molar ratio of $\mathrm{DBH}$ to the substrate 1 were given in the Table), pyrazoline $1(2 \mathrm{mmol})$ and $\mathrm{CCl}_{4}(10 \mathrm{~mL})$ was stirred vigorously magnetically at room temperature. The progress of the reaction was followed by TLC. Reactions were completed after 0.3-4.5 hrs (Table). After the reaction was completed. $\mathrm{K}_{2} \mathrm{CO}_{3}(\mathrm{lg})$ added to the reaction mixture, and the resulting mixture was stirred vigorously magnetically for $0.5 \mathrm{~h}$. Then solid materials were removed by filtration and washing with $\mathrm{CCl}_{4}(10 \mathrm{~mL})$. The solvent was evaporated and the pyrazoles (2) were obtained (Table). If further purification is needed. flash chromatography on silica gel [eluent: $n$-hexane : acetone ( $10: 1)$ ] to give highly pure 2

30. Oxidation of pyrazoline (1c) to pyrazole (2c) with DBH under solventfree condition. A typical procedure. A mixture of compound $1 \mathrm{c}(596 \mathrm{~g} .2 .0 \mathrm{mmol})$ and $\mathrm{DBH}(1.429 \mathrm{~g} .5 \mathrm{mmol})$ was shaken at room temperature for 1.25 hour. After the reaction was completed. $\mathrm{K}_{2} \mathrm{CO}_{3}(\mathrm{lg})$ was added to the reaction mixture. and shaken for $0.5 \mathrm{~h}$. Dichloromethane $(20 \mathrm{~mL})$ was added to the resulting mixture then filtered. Dichloromethane was removed. The vield was $0.491 \mathrm{~g}\left(83^{\circ}, 0\right)$ of erystalline vellow solid (2c). $\mathrm{mp}$ $135-138^{\circ} \mathrm{C}\left[\mathrm{Lit}^{13} \mathrm{mp} 139-140^{\circ} \mathrm{C}\right]$. 